\title{
Stage-specific differential gene expression in Leishmania infantum: from the foregut of Phlebotomus perniciosus to the human phagocyte
}

\author{
Pedro J Alcolea ${ }^{1 *}$, Ana Alonso ${ }^{* *}$, Manuel J Gómez ${ }^{2,4}$, Marina Postigo $^{2}$, Ricardo Molina ${ }^{3}$, Maribel Jiménez ${ }^{3}$
} and Vicente Larraga ${ }^{1}$

\begin{abstract}
Background: Leishmania infantum is the etiological agent of zoonotical visceral leishmaniasis in the Mediterranean basin. A recent outbreak in humans has been recently reported in central Spain. Leishmania spp. parasites are transmitted to the mammalian host by the bite of sand flies. The primary vector of $L$. infantum in Spain is Phlebotomus perniciosus. For decades, research on these parasites has involved the axenic culture model of the promastigote stage including gene expression profiling studies performed in the post-genome era. Unlike the controversial axenic culturing of amastigotes, promastigote cultures are generally accepted and used, although with the precaution of avoiding excessive culture passage.

The primary objective of this differentiation study is to compare the gene expression profiles of promastigotes isolated from the foregut of the sand fly and amastigotes. For this purpose, P. perniciosus sand flies were infected with $L$. infantum and differentiated promastigotes were extracted by dissection of the foreguts. Shotgun DNA microarray hybridization analyses allowed for transcriptome comparison of these promastigotes with amastigotes obtained by infection of the U937 cell line. The results have been compared with those described in published expression analyses using axenic promastigotes.
\end{abstract}

Results: A total of 277 up-regulated genes were found through this hybridization experiment. The comparison of these particular results with published gene expression profile analyses performed using the same experimental procedure to study cultured promastigotes in stationary phase versus amastigotes revealed considerable differences (approximately $95 \%$ of the up-regulated genes were different). We found that the up-regulation rate is lower in amastigotes than in sand fly-derived promastigotes, which is in agreement with the over-expression of genes involved in gene expression regulation and signaling in those promastigote populations.

Conclusions: The up-regulation rate is lower in intracellular amastigotes than in promastigotes obtained from the sand fly gut. This was also reported by us using the promastigote culture model and is an evidence for the hypothesis of promastigote preadaptation towards life in the intracellular environment. Regarding transcript abundance, the set of differentially regulated genes is notably different when using promastigotes from the sand fly foregut instead of axenic cultures.

Keywords: Leishmania infantum, Phlebotomus perniciosus, Promastigotes, Amastigotes, Promastigote axenic culture, Gene expression profiling

\footnotetext{
* Correspondence: pjalcolea@cib.csic.es; amalonso@cib.csic.es

'Laboratorio de Parasitología Molecular, Departamento de Microbiología

Molecular y Biología de las Infecciones, Centro de Investigaciones Biologicas,

Consejo Superior de Investigaciones Científicas, Calle Ramiro de Maeztu, 9.

28040 Madrid, Spain

Full list of author information is available at the end of the article
} 


\section{Background}

Leishmaniasis is a compendium of neglected vector-borne infectious diseases caused by kinetoplastid protozoa of the genus Leishmania with an estimated prevalence of 12 million people worldwide. Visceral leishmaniasis is fatal without treatment and annually leads to 60,000 deaths at least $[1,2]$. L. infantum is the ethiological agent of zoonotic visceral leishmaniasis in the Mediterranean basin and this species also acts as an opportunistic pathogen, as indicated by the increase in co-infections with HIV $[3,4]$. An important outbreak of human leishmaniasis has been reported recently in Fuenlabrada, located in the southwest of the Madrid region [5,6]. The life cycle of the parasite (Figure 1A) is dimorphic and digenetic because the two stages develop in different hosts. Procyclic promastigotes differentiate to metacyclics inside the gut of female sand flies (Diptera: Psychodidae, Phlebotominae), which inject parasites into the mammalian host during blood feeds. Amastigotes survive inside parasitophorous vacuoles of phagocytic mononuclear cells and are able to infect other phagocytes after subsequent proliferation. Phlebotomus perniciosus and P. ariasi are the proven vectors of L. infantum in Spain [7] and P. perniciosus is the major vector of $L$. infantum in the central and western Mediterranean basin [8].

The difficulty of studying promastigotes in their natural environment, the gut of the sand fly, is due to manipulation and reduced biomass. To overcome these problems, axenic cultures of Leishmania spp. promastigotes in liquid media were developed in the $1960 \mathrm{~s}$ and $70 \mathrm{~s}$ in an attempt to reproduce in vitro the conditions inside the gut of the sand fly [9-12]. These media are undefined, as they contain macromolecules, proteins, lipoid substances, trace elements and low molecular weight nutrients. Promastigote cultures are incubated generally at approximately $26-27^{\circ} \mathrm{C}$ also imitating the conditions inside the gut of the sand fly (reviewed by $[13,14]$ ). Promastigotes are maintained in culture for over a week reaching stationary phase and then the death phase, although a considerable proportion of the cells are able to survive for weeks. Compared with the axenic culture model of amastigotes [15,16], the promastigote culture model is stable and reproducible and is widely used for the study of almost all aspects of the biology of this stage in all species of the genus Leishmania. In fact, it is used so routinely that the status of axenically cultured promastigotes has been rarely considered. However, it has been reported that after numerous passages, the original features, infectivity and virulence of the parasite become attenuated, and they frequently require passages through laboratory animals, such as hamsters (reviewed in [13]). Culture passaging does not affect structural studies on specific proteins, as an example, but other research may be affected, such as the evaluation of infectivity, parasitehost cell interactions or the immune response of the host.

The analysis of stage-specific gene regulation in trypanosomatids has provided not only data about the particular expression profiles of hundreds of genes but also valuable information about the biology of these pathogens. First, low stage-specific regulation rates have been described [15-29]. Furthermore, expression profiling confirms that axenically cultured amastigotes are not equivalent to intracellular

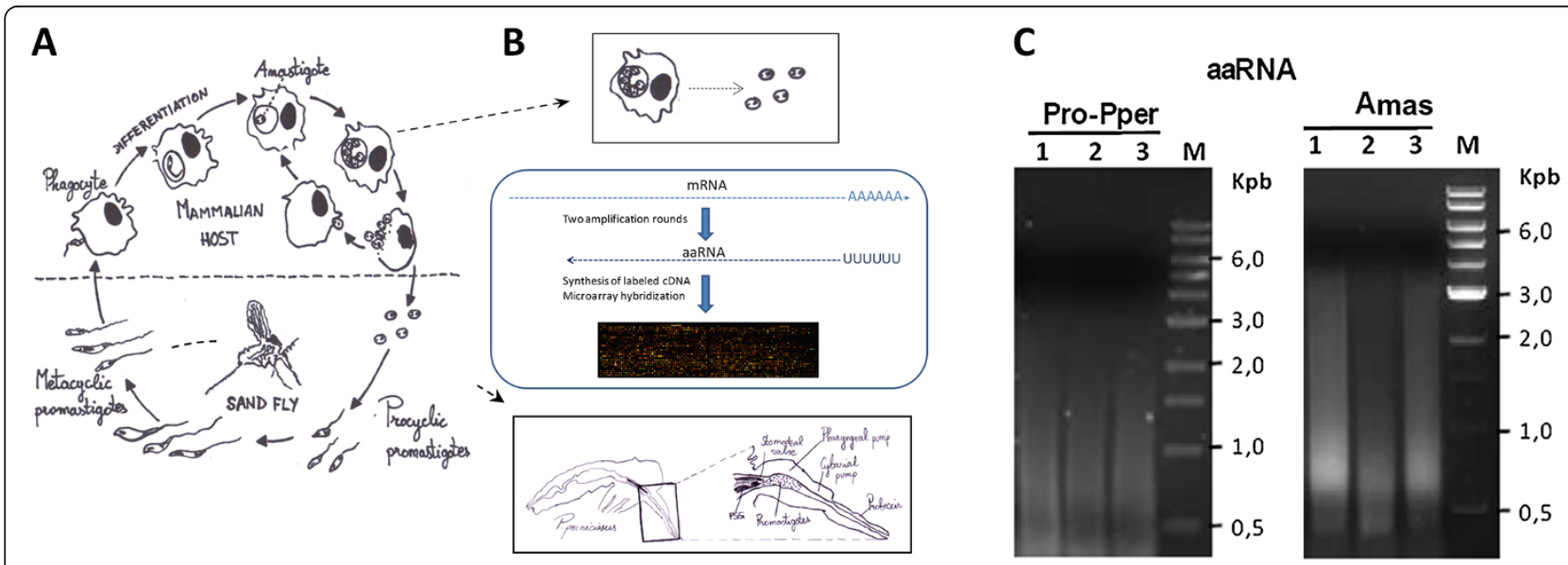

Figure 1 Sampling and mRNA amplification. (A) The life cycle of Leishmania spp. (B) Promastigote RNA extraction was performed immediately after dissection of the sand fly guts and mild lysis of U937 cells. Pro-Pper samples were immediately washed in PBS and lysed with TRIzol ${ }^{\circledR}$ for total RNA extraction. After that, mRNA was doubly amplified (aaRNA) due to sample amount requirements. This included two cycles of reverse transcription (RT) plus second strand cDNA synthesis (combining the use of the Klenow fragment and the RNase H) plus in vitro transcription (IVT). The RT reaction of the first amplification round was performed with a poly-dT primer and the second strand synthesis and the RT reaction of the second amplification round were performed with random hexamer primers, all of which were provided in the MessageAmp ${ }^{\text {TM }}$ aRNA Amplification Kit. Three biological replicates were obtained to perform the subsequent microarray experiment. (C) Electrophoresed aaRNA samples used for the microarray analysis after synthesis of labeled cDNA. 
amastigotes in L. mexicana [21] and in L. infantum [15,16]. Saxena et al. [19] reported that differentiation of $L$. donovani promastigotes to amastigotes is achieved by a succession of transient and permanent changes in gene expression. In addition, we described the up-regulation of genes directly and indirectly related to infectivity in metacyclic $\mathrm{PNA}^{-}$promastigotes in L. infantum [26], found a lower up-regulation rate in amastigotes with respect to promastigotes [23] and more relevance of temperature increase than acidification in the differentiation process of promastigotes to amastigotes, as well as the confluence of both factors leading to an amastigote-like profile [16].

Even though a limited amount of RNA from promastigotes from the sand fly gut anterior to the stomodeal valve can be isolated, a possibility to overcome this limitation is mRNA amplification. However, the small amount of protein extract from this kind of biological samples would not allow performing proteome analyses with the current approaches. Bearing this in mind, we compared the stage-specific gene expression of metacyclic promastigotes and amastigotes in their natural environments for the first time using a high-throughput transcriptome analysis, which revealed noticeable differences between the expression profiles of uncultured and cultured promastigotes when compared to amastigotes.

\section{Methods}

Promastigote culture, in vitro infection of phagocytes and amastigote isolation

The Leishmania infantum isolate MCAN/ES/98/10445 (zymodeme MON-1) was cultured in complete medium containing RPMI 1640 supplemented with L-glutamine (Cambrex, Karlskoga, Sweden), 10\% heat inactivated fetal bovine serum (HIFBS) (Cambrex) and $100 \mu \mathrm{g} / \mathrm{ml}$ streptomycin - $100 \mathrm{IU} / \mathrm{ml}$ penicillin (Cambrex) at $27^{\circ} \mathrm{C}$. They were used in passages 5 to 10 after extraction of the sand fly foregut to perform in vitro infections (see below) of the U937 cell line from human histiocytic leukemia (ATCC ${ }^{\oplus}$ CRL1593.2) [30] and again to feed sand flies to collect metacyclic promastigote samples from the foregut for the microarray analysis. Both sand fly infection steps were carried out following the procedure detailed in the next subsection. In the first case, promastigotes recovered from the foregut were established in NNN medium and subsequently in complete medium until the specified number of passages. Stationary phase promastigotes were harvested at $2000 \mathrm{~g}$ for $10 \mathrm{~min}$.

Cultures of the U937 cell line were carried out at $37^{\circ} \mathrm{C}$, $5 \% \mathrm{CO}_{2}$ in complete medium for $72 \mathrm{~h}$ and centrifuged at $250 \mathrm{~g}$, followed by a $72 \mathrm{~h}$ incubation in complete medium with $20 \mathrm{ng} / \mathrm{ml}$ phorbol 12-myristate 13-acetate (Sigma, Saint Louis, MO) for stimulation [31]. Adhered cells were mildly rinsed with RPMI supplemented with L-glutamine (Cambrex) and recovered by vigorous shaking and in the presence of $0.5 \mathrm{~g} / \mathrm{l}$ trypsin and $0.2 \mathrm{~g} / \mathrm{l}$ EDTA (Cambrex). Trypsin was inactivated with 1 volume of complete medium and phagocytes were harvested. Infections were performed by incubating $20 \times 10^{6}$ promastigotes $/ \mathrm{ml}$ : $10^{6}$ macrophages $/ \mathrm{ml}$ at $37^{\circ} \mathrm{C}$ for $2 \mathrm{~h}$ in complete medium in a water bath and mild shaking every $15 \mathrm{~min}$. After that, the mixture was centrifuged at $250 \mathrm{~g}$ for $10 \mathrm{~min}$ and incubated in complete medium at $37^{\circ} \mathrm{C}, 5 \% \mathrm{CO}_{2}$ for $72 \mathrm{~h}$. After 2 and $16 \mathrm{~h}$, the cultures were rinsed with complete medium. Once phagocytes were detached again, amastigotes were isolated by mild lysis of phagocytes with $0.5 \%$ SDS in RPMI with vigorous agitation for $1 \mathrm{~min}$ followed by centrifugation at $13,000 \mathrm{~g}$ for $1 \mathrm{~min}$ [32]. Aliquots of the amastigote suspension were checked by Giemsa stain and gp63/gp46 immunofluorescence analysis as previously described [23].

\section{Infection of Phlebotomus perniciosus and isolation of promastigotes}

Infected U937 cells were rinsed and detached as described above. Next, they were resuspended at $10^{6}$ cells $/ \mathrm{ml}$ in defibrinated rabbit blood. The mixture was used to feed 150-200 female sand flies of an established colony [33] over a 3-day chicken skin membrane. The sand flies were maintained in a climatic chamber at $27-28^{\circ} \mathrm{C}$, 90-100\% relative humidity, $17 \mathrm{~h}$ light / $7 \mathrm{~h}$ darkness photoperiod and 30\% fructose solution. Promastigote morphology and location inside the gut of a subset of sand flies were evaluated daily by light microscopy. Metacyclic promastigotes anterior to the stomodeal valves (Pro-Pper) were recovered in PBS with a sterile Pasteur pipette [34] from the foregut at the proper times (5-7 days) depending on the previous observations and immediately centrifuged. For this purpose, dissection of the sand flies was performed for extraction of the digestive tracts, which were then split open by pressure with a coverslip. An aliquot was previously recovered for cell counting.

\section{RNA isolation, mRNA amplification and synthesis of labeled cDNA}

Total RNA from three biological replicates of each condition was immediately extracted with $\mathrm{TRizol}^{\circledR}$ reagent (Life Technologies, Carlsbad, CA) following the manufacturer's instructions. The volume of $\mathrm{TRIzol}^{-}$reagent used was $0.5 \mathrm{ml}$ for each of three Pro-Pper replicates and $1 \mathrm{ml}$ for amastigote samples. Glycogen at $1 \mu \mathrm{g} / \mathrm{ml}$ (Life Technologies) was used as carrier prior to 2-propanol precipitation in the total RNA isolation procedure of Pro-Pper samples. RNA quality was assessed with an Experion RNA HighSens Analysis Kit (Bio-Rad Laboratories, Hercules, CA) and conventional agarose gel electrophoresis. Thereafter, two mRNA amplification rounds were performed with MessageAmp ${ }^{\text {TM }}$ II aRNA Amplification Kit 
(Life Technologies) as previously described [23] thus yielding antisense doubly amplified RNA (aaRNA). The integrity of aRNA and aaRNA samples was checked by $1 \%$ agarose gel electrophoresis.

The first strand aminoallyl-cDNA was synthesized. First, denaturing of $10 \mu \mathrm{g}$ of aaRNA together with $6 \mu \mathrm{g}$ of random primers (Life Technologies) was carried out by incubation at $70^{\circ} \mathrm{C}$ for $10 \mathrm{~min}$ and snap-chill on ice. Then, samples were incubated at $46^{\circ} \mathrm{C}$ for $3 \mathrm{~h}$ with $570 \mu \mathrm{M}$ each dATP, dCTP, dGTP, $230 \mu \mathrm{M}$ dTTP, $340 \mu \mathrm{M}$ aminoallyl-dUTP, $10 \mu \mathrm{M}$ DTT and $600 \mathrm{U}$ SuperScript ${ }^{\oplus}$ Reverse Transcriptase (Life Technologies) in a $30 \mu \mathrm{l}$ final volume. Then, a $70^{\circ} \mathrm{C}, 30 \mathrm{~min}$ incubation in $100 \mathrm{mM} \mathrm{NaOH} / 10 \mathrm{mM}$ EDTA allowed DNA degradation. After neutralization with $3 \mu \mathrm{l}$ of $3 \mathrm{M}$ sodium acetate $\mathrm{pH}$ 5.2, single stranded cDNA samples were purified with QiaQuick PCR Purification Kit (Qiagen, Hilden, Germany) using phosphate wash buffer ( $5 \mathrm{mM} \mathrm{KPO}_{4}, 80 \%$ ethanol, $\mathrm{pH}$ 8.0) and phosphate elution buffer (4 $\left.\mathrm{mM}^{\mathrm{K}} \mathrm{KOO}_{4}\right)$ instead of the wash and elution buffers provided in the kit. Next, samples were completely dried in a vacuum centrifuge and resuspended in $10 \mu \mathrm{l}$ of water, mixed with $5 \mu \mathrm{l}$ of $12 \mathrm{ng} / \mu \mathrm{l}$ DMSO-dissolved Cy3 or Cy5 monofunctional dye (respectively for amastigotes and promastigotes) (GE Healthcare, Chalfont Saint Giles, UK) and incubated at room temperature in darkness for $1 \mathrm{~h}$ for coupling with the aminoallyl residues. Labeled cDNA samples were then purified with a QiaQuick PCR Purification Kit (Qiagen) entirely following the manufacturer's instructions.

\section{Microarray hybridization and analysis of data}

The construction of the complete shotgun genomic DNA microarrays of $L$. infantum used has been published [26] and deposited in the GEO repository supplying MIAME compliant data (http://www.ncbi.nlm.nih.gov/geo/ query/acc.cgi?acc=GSE11269). Prior to hybridization, the microarrays were soaked first in $0.1 \% \mathrm{~N}$-lauroylsarcosine in $2 \mathrm{xSC}$, then soaked in $2 \mathrm{xSSC}$ and then denatured at $95^{\circ} \mathrm{C}$ for $3 \mathrm{~min}$, fixed in chilled 100\% ethanol and spun dry in a slide mini centrifuge. The microarrays were blocked by attachment upside down to a $60 \mathrm{ml}$ drop of $3 \mathrm{x}$ SSC, $0.3 \%$ N-lauroylsarcosine, $60 \mathrm{mM}$ Tris- $\mathrm{HCl} \mathrm{pH} 8.0,83 \mathrm{ng} / \mathrm{ml}$ denatured herring sperm DNA and 1\% BSA over a Hybri-Slip coverslip (Sigma) and incubated at $42^{\circ} \mathrm{C}$ in a water bath for $30 \mathrm{~min}$. Then, labeled cDNA samples were mixed in equimolar amounts of each dye $(50 \mathrm{pmol})$ and incubated at $40^{\circ} \mathrm{C}$ with blocked microarrays for $16 \mathrm{~h}$ (same composition of blocking solution except for $0.1 \%$ BSA, $25 \mathrm{ng} / \mathrm{ml}$ poly (T), 50\% deionized formamide). After that, the slides were soaked in $2 x \mathrm{SSC}, 0.2 \%$ SDS at $40^{\circ} \mathrm{C}$ and consecutively in $1 \mathrm{x}$ SSC and $0.2 \mathrm{x}$ SSC at room temperature.

Genomic DNA was isolated from non-infected sand flies and U937 cells by phenolic extraction as described previously [26] and directly labeled with
Cy5 $(350 \mu \mathrm{M}$ each dATP, dCTP, dGTP and (1/3 Cy5-dUTP, 2/3 dTTP) mix) using GenomiPhi ${ }^{\mathrm{TM}}$ DNA Amplification Kit (GE Healthcare). Single dye hybridizations with $L$. infantum DNA microarrays were performed as a cross-hybridization control.

The hybridized slides were scanned with a GenePix 4100A instrument (Axon, Foster City, CA) and raw data with local feature background medians subtracted were obtained with GenePix Pro 7.0 software. Normalization with the LOWESS per pin algorithm and statistical inference using the paired t-test and FDR adjustment were performed with AlmaZen software (BioAlma, Tres Cantos, Spain) and checked with the TIGR Multi Experiment Viewer 4.3. The cutoff values were the following: (i) fold change $\mathrm{F} \geq 2(\mathrm{Cy} 5 / \mathrm{Cy} 3$ ratio if $\mathrm{Cy} 5>\mathrm{Cy} 3)$ or $\mathrm{F} \leq-2(-\mathrm{Cy} 3 / \mathrm{Cy} 5$ ratio if $\mathrm{Cy} 3>\mathrm{Cy} 5)$, (ii) total relative fluorescence intensity value $>5000$ arbitrary fluorescence units and (iii) $\mathrm{p}^{*}<0.05$. Three replicates were considered in the experiment.

\section{Identification of stage-regulated genes}

The insert ends of clones that fulfilled the cutoff values mentioned were recovered from the genomic library used for microarray construction, sequenced with the M13-pUC18 primers and assembled as described, a strategy that is not affected by insertions, deletions and substitutions between the MCAN/ES/98/10445 and the genome-sequenced MCAN/ES/98/LLM-877 isolates [26]. The conditions used to consider the sequence of a given clone assembled were: (i) e-value $<1$ e-10 for both ends, (ii) convergent orientation in the genome sequence and (iii) length $\leq 11 \mathrm{kbp}$, according to the features of the genome library [26]. The analyzed clones were classified in three categories according to the fulfillment of such conditions: in $a$ clones, only one pair of alignments complies with all three conditions; in $b$ clones, more than one pair does due to adjoining sequence repeats and is therefore the best sequence identity; and $c$ clones do not fulfill the requirements to be assembled for unpaired alignment or incongruent pair of alignments presumably due to the presence of two or more inserts in the clone. Once clones were assembled, identification of genes overlapping with them was performed using a Perl script with a 5\% overlapping length cutoff. Clones that do not fulfill this criterion but align with less than $5 \%$ of the length of a given annotated ORF were identified using the genome browser [26]. Those clones that do not map with any ORF were aligned with complete transcript sequences including UTRs that were obtained by RNAseq in L. major [35]. Gene sequences were analyzed with BLAST2GO [36] to classify them in functional categories. In addition, the search of all genes in literature and the databases GeneDB [37], TriTrypDB [38] and KEGG [39] provided further functional information. CLUSTALW2 alignments 
allowed distinguishing gene copies from genes encoding isoforms.

\section{Real time quantitative RT-PCR (qRT-PCR) validation}

Unlabelled single stranded cDNA was synthesized following the same procedure described above but using a mixture stock of $10 \mathrm{mM}$ of each dNTP. Custom TaqMan MGB Assays-by-Design (specifically FAM-NFQ MGB probes) (Life Technologies) were run in a $7900 \mathrm{HT}$ Fast Real Time PCR system (Life Technologies) using TaqMan $^{\odot}$ Universal Master Mix 2x (Life Technologies) following the manufacturer's instructions. Thermal cycling was as follows: $95^{\circ} \mathrm{C}$ for $5 \mathrm{~min}, 40$ cycles $\left[95^{\circ} \mathrm{C}\right.$ for $30^{\prime}, 60^{\circ} \mathrm{C}$ for $1 \mathrm{~min}$ ]. PCR efficiencies were calculated by the standard curve best fit method from a triplicate dilution series experiment for each gene and cDNA sample (Pro-Pper/Amastigotes). Coefficients of variation were previously checked. Fold changes were calculated with respective efficiency-corrected normalized quantities in the same fashion as for microarray data. Normalized quantities were calculated by dividing the raw quantity value (efficiency to the power of $-\mathrm{Ct}$ ) of the gene of interest by that of the endogenous control (GAPDH gene of L. infantum). Sequences of primers and probes are listed in the Additional file 1.

\section{Binomial test and hierarchical clustering}

A binomial test was performed to infer the level of significance of the differences in absolute frequencies of up-regulated and down-regulated genes in Pro-Pper/A as previously described [23]. An iterative hierarchical clustering analysis was also carried out with TIGR's MultiExperiment Viewer 4.3 (MEV) by introducing normalized microarray hybridization data matrixes (including medians and standard deviations of intensity and $F$ values) of clones with significant differential regulation in the experiment reported herein and the previously available data describing differential gene expression profiles of cultured amastigotes and amastigote-like forms [16,23]. The SAM p-value cutoff was 0.05 , which was the same as for the previous independent t-tests for each experiment. HCL-ST was performed independently for significant and non-significant genes. ST algorithm with a jackknifing resampling option and 100 iterations for the construction and clustering of the gene expression matrix were applied in a HCL-ST analysis.

\section{Results and discussion}

mRNA amplification and microarray hybridization analysis of metacyclic promastigotes isolated from $P$. perniciosus and amastigotes

The total amounts of RNA obtained from Pro-Pper replicates were comprised between 20 and $25 \mathrm{ng}$ and after the first amplification round, 200-250 ng of aRNA were obtained. Double amplification of mRNA made the microarray hybridization experiments possible. Obviously RNA samples from amastigotes were treated identically. Electrophoretic analyses of the aaRNA samples including replicates are shown in Figure 1. The number of differences in gene expression found between Pro-Pper and amastigotes is 277 (Figure 2, Table 1), which is comparable to stage-specific gene expression regulation between logarithmic phase promastigotes and amastigotes and higher than between stationary phase promastigotes and amastigotes [23]. According to the 5\% of clone-to-ORF overlapping length cutoff performed with a Perl script (see Methods section) [16,23-26], 143 out of 277 differences correspond to genes of known function or hypothetical proteins genes. The 134 clones (48\%) that do not fulfill this criterion (Table 1) are described in the Additional file 2. Some of them are aligned with less than $5 \%$ of the length of an ORF. The rest of clones do not align with any ORF but presumably do with untranslated regions (UTRs). For this reason, they were aligned against complete transcript sequences of $L$. major including UTRs that were obtained by RNAseq [35]. About half of the Leishmania spp. genes code for hypothetical proteins and proteins of unknown function $[37,38,40,41]$ and this is reflected in the relatively high number of such proteins that are differentially regulated (Table 1, Additional file 2). These facts enable the possibility of extracting additional information from the genome and the transcriptome of these parasites. Redundancy in representation of genome sequences by the genomic library generated for microarray construction [26] is reflected in stage-specific gene expression results because some clones represent the same differentially regulated gene (Table 2). This is an internal validation together with the control spots included in the microarrays [26] (Additional file 3).

\section{qRT-PCR validation}

This approach has been useful not only for the validation of microarray results, but also to sort out the differentially regulated genes in clones fulfilling the cutoff values in the microarray hybridization analysis that align with more than one gene. All these data are reflected in Table 2 and according to them, five genes of known function already resolved by microarrays themselves have been confirmed by qRT-PCR and 16 clones not directly resolved by microarray analysis contain at least one differentially regulated gene. Constant expression values for a given CDS have been obtained only in clones that overlap with more than one CDS. The remaining gene is presumed to be differentially regulated except if more than two CDS overlap with the clone. Consequently, $7.8 \%$ of differentially regulated genes have been validated and we have not detected any differing result between the techniques so far, including those in previous studies [16,23-26]. 


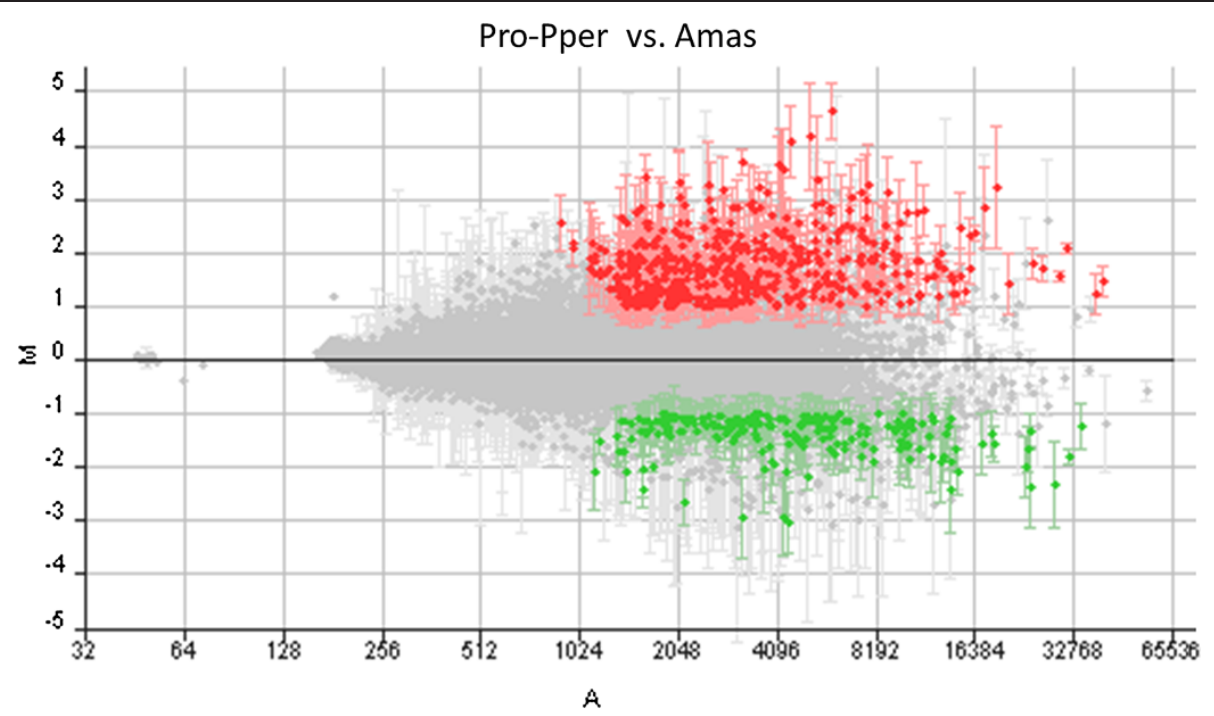

Figure 2 General outcome of the Pro-Pper/A microarray hybridization experiment in L. infantum. M/A scatter plot of hybridization outcomes of all clones fulfilling (highlighted) or not the conditions necessary for containing differentially regulated genes between Pro-Pper and Amas. $\mathrm{M}=\left(\log _{2} \mathrm{Ri}-\log _{2} \mathrm{Gi}\right)$ and $\mathrm{A}=\left[\left(\log _{2} \mathrm{Ri}+\log _{2} \mathrm{Gi}\right) / 2\right]$, where $\mathrm{R}$ and $\mathrm{G}$ are, respectively, red (Cy5) and green (Cy3) fluorescence intensity values. Red spots correspond to selected DNA fragments containing a gene up-regulated by at least 2-fold and green spots represent those down-regulated by at least 2-fold times. Further criteria for spot selection are detailed in the Methods section.

\section{Differential gene expression between Pro-Pper and amastigotes}

The differences found in molecular functions and biological processes are summarized along with the outcome of the BLAST2GO analysis (Figure 3) and the schema based on information from the described analysis, including the cell component terms, literature and GeneDB, TriTrypDB and KEGG [39] databases (Figure 4). Processes related to DNA metabolism, chromosome organization, translation, cellular response to stimulus and stress, transport and movement are associated with up-regulated genes in Pro-Pper with respect to amastigotes (Figure 3). Overall, these data suggest a more active metabolic status of promastigotes, which

Table 1 Overview of the Pro-Pper/A differential gene expression profiles

\begin{tabular}{lcc}
\hline Annotation status & \multicolumn{2}{c}{$\begin{array}{c}\text { Frequency of differentially } \\
\text { regulated genes in Pro-Pper/A }\end{array}$} \\
\cline { 2 - 3 } & Up-regulated & Down-regulated \\
\hline Genes of known function & 46 & 19 \\
Conserved hypothetical protein & 48 & 25 \\
Hypothetical protein & 4 & 1 \\
Clones overlapping with UTRs or & 86 & 48 \\
less than 5\% of an ORF & & 93 \\
(Additional file 2: Table S2) & & \\
Total ( $\mathrm{n}=277$ ) & 184 & \\
\hline Absolute frequncies of genes & & \\
\hline
\end{tabular}

Absolute frequencies of genes encoding for proteins of known function and hypothetical proteins are provided. The frequencies of up-regulated and down-regulated genes have been contrasted with the binomial test. is in agreement with previously reported data [23]. Table 2 contains stage-specific regulated genes of known function and the differentially regulated hypothetical protein genes are included in the Additional file 4. Regarding this transcriptome variation (Figure 4), significant changes in metabolism may take place between promastigotes from the anterior gut of $P$. perniciosus and intracellular amastigotes. The biotin/lipoate ligase genes LinJ.31.1070 and LinJ.36.3230 (BLPL) are over-expressed in Pro-Pper, which suggests an increased demand for lipoic acid and/or biotin by any dehydrogenase complex and/or carboxylase, respectively. In fact, the genes encoding BLPLs bear the activity EC 6.3.4.15 and the activities 6.3.4.9., 6.3.4.10 and 6.3.4.11 are absent in L. infantum (KEGG database). This suggests an important biological role of BLPLs in these parasites. One hypothesis for the central role of this protein in the Pro-Pper/A scenario is highlighted in Figure 4. One of the enzymes demanding the cofactor could be the glycosomal phosphoenolpyruvate carboxykinase (gPEPCK). Provided that the level of gPEPCK transcripts are higher in amastigotes, the expression profile of BLPL may not specially favor gluconeogenesis in Pro-Pper. L. donovani amastigotes also over-express gPEPCK with respect to cultured promastigotes [29]. Another possibility may be increased activity of carboxylases participating in leucine and isoleucine degradation in Pro-Pper but again this is not likely to occur provided up-regulation of the $\alpha$-ketoisovalerate dehydrogenase gene (KIVDH) in amastigotes. This gene was also found to be up-regulated at the protein level in mature L. donovani amastigotes [29]. As a consequence, 
Table 2 Genes of known function that are differentially regulated in Pro-Pper with respect to amastigotes

\begin{tabular}{|c|c|c|c|c|c|c|c|c|c|c|}
\hline \multirow[t]{2}{*}{ Clone } & \multirow[t]{2}{*}{$F$} & \multirow[t]{2}{*}{$\log _{2} R \pm S$} & \multirow[t]{2}{*}{$p$} & \multicolumn{2}{|c|}{ e-value } & \multirow[t]{2}{*}{ Def. } & \multirow[t]{2}{*}{ Annotation } & \multirow[t]{2}{*}{ Annotated gene function } & \multicolumn{2}{|r|}{$q R T-P C R$} \\
\hline & & & & Fw & $R v$ & & & & & \\
\hline $\operatorname{Lin} 13 \mathrm{C} 3$ & 2.72 & $1.4 \pm 0.3$ & 0.011 & 0 & 0 & $b$ & LinJ.21.0770 & $\begin{array}{l}\text { Ribonuclease-L inhibitor, ABC subfamily } \\
\text { E, putative }\end{array}$ & & N.D. \\
\hline Lin16F1 & 3.69 & $1.9 \pm 0.4$ & 0.014 & - & 0 & c & LinJ.23.0050 & Peroxidoxin/tryparredoxin peroxidase & & N.D. \\
\hline \multirow[t]{2}{*}{ Lin16C2 } & 24.97 & $4.6 \pm 0.5$ & 0.004 & 0 & 0 & $b$ & LinJ.35.3930 & EF-hand calmodulin-like protein & + & $65.3 \pm 3.1$ \\
\hline & & & & & & & LinJ.35.3940 & Hypothetical protein, conserved & & N.D. \\
\hline Lin17G12 & 2.49 & $1.3 \pm 0.4$ & 0.034 & 0 & 0 & a & LinJ.19.0940 & 4-coumarate-CoA ligase & & N.D. \\
\hline $\mathrm{Lin} 21 \mathrm{H} 10$ & 17.97 & $4.2 \pm 1.0$ & 0.019 & 0 & 0 & b & LinJ.26.1670 & Sphingolipid $\delta$-4 desaturase, putative & + & $4.1 \pm 0.3$ \\
\hline Lin22C9 & 2.14 & $1.1 \pm 0.4$ & 0.040 & 0 & 0 & b & LinJ.33.2910 & Ubiquitin-conjugating enzyme, putative & & N.D. \\
\hline $27 \mathrm{C} 6$ & 4.17 & $2.1 \pm 0.2$ & 0.002 & 0 & 0 & a & LinJ.31.1240 & $\begin{array}{l}\text { Vacuolar } \mathrm{H}^{+} \text {-translocating pyrophosphatase, } \\
\text { putative }\end{array}$ & & N.D. \\
\hline Lin28C5 & 7.31 & $2.9 \pm 0.6$ & 0.020 & 0 & 0 & $b$ & LinJ.26.1670 & Sphingolipid $\delta$-4 desaturase, putative & + & $4.1 \pm 0.3$ \\
\hline Lin31D11 & 3.00 & $1.6 \pm 0.3$ & 0.016 & 0 & 0 & $\mathrm{~b}$ & LinJ.31.1870 & Protein kinase-like protein & & N.D. \\
\hline \multirow[t]{4}{*}{ Lin34F1 } & 2.98 & $1.6 \pm 0.5$ & 0.038 & & & & LinJ.08.1000 & Histone deacetylase, putative & & N.D. \\
\hline & & & & & & & LinJ.26.1620 & 40 S ribosomal protein $\$ 33$, putative & + & $92.2 \pm 5.2$ \\
\hline & & & & & & & LinJ.26.1630 & 40 S ribosomal protein $\$ 33$, putative & + & $92.2 \pm 5.2$ \\
\hline & & & & & & & LinJ.26.1640 & Hypothetical protein, conserved & & N.D. \\
\hline Lin41C12 & 2.95 & $1.6 \pm 0.5$ & 0.028 & 0 & 0 & $b$ & LinJ.31.1600 & Cytochrome c oxidase VIII (coxVIII), putative & & N.D. \\
\hline Lin45A11 & 2.35 & $1.2 \pm 0.4$ & 0.033 & 0 & 0 & b & LinJ.28.2220 & Mitochondrial DEAD protein, putative & & N.D. \\
\hline Lin48B6 & 2.60 & $1.4 \pm 0.4$ & 0.026 & 0 & 0 & b & LinJ.36.2050 & Mismatch repair protein MSH8, putative & & N.D. \\
\hline Lin49B7 & 9.21 & $3.2 \pm 1.1$ & 0.039 & 0 & 0 & $b$ & LinJ.06.1320 & Pteridin transporter, putative & & N.D. \\
\hline Lin50G5 & 3.83 & $1.9 \pm 0.4$ & 0.016 & 0 & 0 & a & LinJ.21.2080 & Cytochrome oxidase VI (coxVl), putative & & N.D. \\
\hline Lin51A8 & 2.24 & $1.2 \pm 0.4$ & 0.041 & 0 & 0 & a & LinJ.32.4190 & GIPL-galf transferase, putative & & N.D. \\
\hline Lin51E2 & 2.09 & $1.1 \pm 0.2$ & 0.018 & 0 & 0 & b & LinJ.36.0020 & Histone $\mathrm{H} 4$ & & N.D. \\
\hline \multirow[t]{2}{*}{ Lin51G7 } & 3.85 & $1.9 \pm 0.2$ & 0.003 & 0 & 0 & b & LinJ.19.1490 & Oxidoreductase-like protein & + & $3.6 \pm 0.2$ \\
\hline & & & & & & & LinJ.19.1500 & Hypothetical protein, conserved & & N.D. \\
\hline \multirow[t]{3}{*}{ Lin54C2 } & 7.13 & $2.8 \pm 0.5$ & 0.010 & 0 & 0 & a & LinJ.06.1310 & Mitogen-activated protein kinase & + & $10.8 \pm 0.5$ \\
\hline & & & & & & & LinJ.06.1320 & Hypothetical protein, conserved & & N.D. \\
\hline & & & & & & & LinJ.06.1330 & Hypothetical protein, conserved & & N.D. \\
\hline Lin58H6 & 5.43 & $2.4 \pm 1.0$ & 0.049 & 0 & 0 & $\mathrm{~b}$ & LinJ.08.0030 & $\begin{array}{l}\text { Vesicle-associated membrane protein, } \\
\text { putative }\end{array}$ & & N.D. \\
\hline $\mathrm{Lin} 60 \mathrm{H} 10$ & 4.41 & $2.1 \pm 0.2$ & 0.003 & 0 & 0 & a & LinJ.23.0630 & Oxidoreductase-like protein & + & $2.9 \pm 0.1$ \\
\hline Lin76A1 & 3.08 & $1.6 \pm 0.4$ & 0.018 & 0 & 0 & a & LinJ.31.3320 & Histone $\mathrm{H} 4$ & + & $11.0 \pm 0.4$ \\
\hline Lin76F1 & 5.40 & $2.4 \pm 0.5$ & 0.013 & 0 & 0 & $b$ & LinJ.34.3370 & Phosphatidylinositol 4-kinase, putative & & N.D. \\
\hline Lin77B12 & 2.01 & $1.0 \pm 0.3$ & 0.038 & 0 & 0 & a & LinJ.27.1520 & $\begin{array}{l}\text { Eukaryotic translation initiation factor elF4E, } \\
\text { putative }\end{array}$ & & N.D. \\
\hline Lin80B3 & 2.83 & $1.5 \pm 0.6$ & 0.049 & 0 & 0 & $b$ & LinJ.28.3250 & $\begin{array}{l}\text { Glucose-6-phosphate N-acetyltransferase, } \\
\text { putative }\end{array}$ & & N.D. \\
\hline \multirow[t]{3}{*}{ Lin82D10 } & 4.25 & $2.1 \pm 0.5$ & 0.019 & 0 & 0 & a & LinJ.23.0040 & $\beta$-propeller, putative & + & $2.0 \pm 0.1$ \\
\hline & & & & & & & LinJ.23.0050 & Peroxidoxin/Tryparedoxin peroxidase & + & $21.2 \pm 0.8$ \\
\hline & & & & & & & LinJ.23.0060 & Cyclophilin, putative & & N.D. \\
\hline Lin89D8 & 2.87 & $1.5 \pm 0.4$ & 0.023 & 0 & 0 & a & LinJ.36.3230 & Lipoate protein ligase, putative & + & $8.0 \pm 0.5$ \\
\hline \multirow[t]{2}{*}{ Lin93D6 } & 5.50 & $2.5 \pm 0.3$ & 0.005 & 0 & 0 & b & LinJ.26.1670/80 & Sphingolipid $\delta-4$ desaturase, putative & + & $4.1 \pm 0.4$ \\
\hline & & & & & & & LinJ.26.1690 & $\begin{array}{l}\text { Cytochrome c oxidase, subunit V (coxV), } \\
\text { putative }\end{array}$ & & N.D. \\
\hline Lin96H7 & 4.92 & $2.3 \pm 0.3$ & 0.007 & 0 & 0 & $\mathrm{a}$ & LinJ.31.3310 & Hypothetical protein, unknown function & & N.D. \\
\hline
\end{tabular}


Table 2 Genes of known function that are differentially regulated in Pro-Pper with respect to amastigotes (Continued)

\begin{tabular}{|c|c|c|c|c|c|c|c|c|c|c|}
\hline & & & & & & & LinJ.31.3320 & Histone $\mathrm{H} 4$, putative & + & $11.0 \pm 0.4$ \\
\hline \multirow[t]{2}{*}{ Lin96B8 } & 4.21 & $2.1 \pm 0.8$ & 0.046 & 0 & 0 & a & LinJ.31.3310 & Hypothetical protein, unknown function & & N.D. \\
\hline & & & & & & & LinJ.31.3320 & Histone $\mathrm{H} 4$, putative & + & $11.0 \pm 0.4$ \\
\hline Lin99G6 & 4.02 & $2.0 \pm 0.5$ & 0.002 & $4 e-156$ & $9 e-80$ & $b$ & LinJ.36.1730 & Proteasome subunit $\beta 5$, putative & + & $5.2 \pm 0.2$ \\
\hline Lin105H8 & 3.92 & $2.0 \pm 0.6$ & 0.026 & 0 & 0 & $b$ & LinJ.36.3750 & Cysteine synthase, putative & + & $3.6 \pm 0.0$ \\
\hline Lin106G3 & 2.26 & $1.2 \pm 0.3$ & 0.029 & 0 & 0 & a & LinJ.31.1070 & Biotin/lipoate-protein ligase & + & $8.0 \pm 0.5$ \\
\hline Lin110F5 & 3.55 & $1.8 \pm 0.3$ & 0.011 & 0 & 0 & a & LinJ.16.1220 & $60 S$ ribosomal protein $\mathrm{L} 39$, putative & & N.D. \\
\hline Lin111D8 & 9.80 & $3.3 \pm 0.8$ & 0.020 & 0 & 0 & a & LinJ.08.1000 & Histone deacetylase, putative & & N.D. \\
\hline \multirow[t]{3}{*}{ Lin113B9 } & 2.72 & $1.4 \pm 0.6$ & 0.048 & 0 & 0 & a & LinJ.36.0550 & Hypothetical protein, conserved & & N.D. \\
\hline & & & & & & & LinJ.36.0560 & Protein phosphatase $2 \mathrm{C}$, putative & + & $6.4 \pm 0.2$ \\
\hline & & & & & & & LinJ.36.0570 & Small nuclear ribonucleoprotein, putative & + & $9.4 \pm 0.8$ \\
\hline Lin125F11 & 5.88 & $2.5 \pm 1.0$ & 0.046 & $7 e-56$ & $3 e-55$ & a & LinJ.32.2780 & Cistathionine $\gamma$-liase, putative & & N.D. \\
\hline \multirow[t]{4}{*}{ Lin130C5 } & 2.52 & $1.3 \pm 0.5$ & 0.040 & $3 e-178$ & 0 & $b$ & LinJ.36.3170 & Exosome exonuclease RRP41, putative & - & $-3.3 \pm 0.2$ \\
\hline & & & & & & & LinJ.36.3180 & Clathrin coat assembly protein & & N.D. \\
\hline & & & & & & & LinJ.36.3190 & Pre-mRNA branch site p14 protein, putative & + & $43.2 \pm 1.3$ \\
\hline & & & & & & & LinJ.36.3200 & Hypothetical protein, conserved & & N.D. \\
\hline Lin132A11 & 5.24 & $2.4 \pm 0.1$ & 0.001 & 0 & 0 & a & LinJ.31.1240 & $\begin{array}{l}\text { Vacuolar } \mathrm{H}^{+} \text {-translocating pyrophosphatase, } \\
\text { putative }\end{array}$ & & N.D. \\
\hline \multirow[t]{2}{*}{ Lin136G4 } & 2.66 & $1.4 \pm 0.6$ & 0.049 & 0 & 0 & $b$ & LinJ.22.1360 & Hypothetical protein, conserved & & N.D. \\
\hline & & & & & & & LinJ.22.1370 & 605 ribosomal protein L14, putative & + & $4.1 \pm 0.3$ \\
\hline Lin139D8 & 5.85 & $2.5 \pm 0.6$ & 0.003 & 0 & 0 & $b$ & LinJ.08.0010 & $\begin{array}{l}\text { Structural maintenance of chromosome } \\
\text { protein } 3 \text {, putative }\end{array}$ & & N.D. \\
\hline \multirow[t]{2}{*}{ Lin146A12 } & 2.67 & $1.4 \pm 0.4$ & 0.032 & 0 & 0 & $b$ & LinJ.30.0710 & $40 \mathrm{~S}$ ribosomal protein $\mathrm{S30}$, putative & + & $56.2 \pm 1.7$ \\
\hline & & & & & & & LinJ.30.0720 & NUDC-like protein & & N.D. \\
\hline Lin166F2 & 5.54 & $2.5 \pm 0.7$ & 0.023 & 0 & 0 & $b$ & LinJ.21.0770 & $\begin{array}{l}\text { Ribonuclease }-L \text { inhibitor, } A B C \text { subfamily } \\
\text { E, putative }\end{array}$ & & N.D. \\
\hline \multirow[t]{2}{*}{ Lin166H10 } & 2.08 & $1.1 \pm 0.2$ & 0.014 & 0 & 0 & b & LinJ.26.1680 & Sphingolipid $\delta-4$ desaturase, putative & + & $4.1 \pm 0.4$ \\
\hline & & & & & & & LinJ.26.1690 & Cytochrome b5 reductase, putative & & N.D. \\
\hline Lin168F2 & 2.17 & $1.1 \pm 0.2$ & 0.017 & 0 & 0 & a & LinJ.32.0710 & OSM-3-like kinesin & & N.D. \\
\hline Lin169E6 & 6.66 & $2.7 \pm 0.9$ & 0.037 & 0 & 0 & $b$ & LinJ.32.0550 & Profilin, putative & & N.D. \\
\hline \multirow[t]{2}{*}{ Lin172B9 } & 4.42 & $2.1 \pm 0.4$ & 0.009 & 0 & 0 & $b$ & LinJ.26.1680 & Sphingolipid $\delta-4$ desaturase, putative & + & $4.1 \pm 0.4$ \\
\hline & & & & & & & LinJ.26.1690 & Cytochrome b5 reductase, putative & & N.D. \\
\hline Lin208F7 & 3.33 & $1.7 \pm 0.6$ & 0.033 & 0 & 0 & $b$ & LinJ.30.3640 & Ser/Thr protein kinase, putative & & N.D. \\
\hline Lin276F6 & 3.47 & $1.8 \pm 0.3$ & 0.007 & 0 & 0 & $b$ & LinJ.35.2370 & Protein kinase, putative & & N.D. \\
\hline Lin290F2 & 3.52 & $1.8 \pm 0.3$ & 0.012 & 0 & 0 & $b$ & LinJ.04.1250 & Actin & & N.D. \\
\hline $\mathrm{Lin} 298 \mathrm{H} 2$ & 7.23 & $2.8 \pm 0.5$ & 0.011 & 0 & 0 & $b$ & LinJ.22.1340 & Ser/Thr protein phosphatase & & N.D. \\
\hline Lin299A1 & 4.34 & $2.1 \pm 0.8$ & 0.045 & 0 & 0 & $b$ & LinJ.36.1720 & $\begin{array}{l}\text { Universal minicircle sequence binding } \\
\text { protein (UMSBP), putative }\end{array}$ & & N.D. \\
\hline $\operatorname{Lin} 18 \mathrm{~A} 12$ & -2.20 & $-1.1 \pm 0.4$ & 0.044 & 0 & 0 & $b$ & LinJ.33.2430 & UDP-glucose 4'-epimerase & & N.D. \\
\hline Lin25B7 & -2.34 & $-1.2 \pm 0.4$ & 0.034 & 0 & 0 & $b$ & LinJ.31.3390 & Sodium stibogluconate resistance protein & & N.D. \\
\hline $\mathrm{Lin} 30 \mathrm{H} 4$ & -3.45 & $-1.8 \pm 0.4$ & 0.017 & 0 & $2 e-111$ & b & LinJ.27.2500 & $\begin{array}{l}\text { Glycosomal phosphoenolpyruvate } \\
\text { carboxykinase, putative }\end{array}$ & & N.D. \\
\hline Lin35H4 & -2.77 & $-1.5 \pm 0.5$ & 0.039 & 0 & 0 & $b$ & LinJ.34.3740 & $\begin{array}{l}\text { Expression-site associated glycoprotein } \\
\text { (ESAG5), putative }\end{array}$ & & N.D. \\
\hline Lin49D6 & -2.83 & $-1.5 \pm 0.5$ & 0.031 & 0 & $1 e-152$ & $b$ & LinJ.19.0590 & Protein kinase, putative & & N.D. \\
\hline Lin54A3 & -2.21 & $-1.1 \pm 0.4$ & 0.037 & $4 \mathrm{e}-156$ & 0 & b & LinJ.36.6510 & Small G protein, putative & & N.D. \\
\hline Lin77H8 & -2.07 & $-1.0 \pm 0.4$ & 0.039 & 0 & 0 & $b$ & LinJ.08.0690 & Amastin-like protein & & N.D. \\
\hline
\end{tabular}


Table 2 Genes of known function that are differentially regulated in Pro-Pper with respect to amastigotes (Continued)

\begin{tabular}{|c|c|c|c|c|c|c|c|c|c|c|}
\hline Lin88B2 & -2.08 & $-1.1 \pm 0.4$ & 0.040 & 0 & 0 & $b$ & LinJ.10.1070 & Histone $\mathrm{H} 3$ & & N.D. \\
\hline Lin101D5 & -2.54 & $-1.3 \pm 0.3$ & 0.017 & 0 & $2 e-28$ & $b$ & LinJ.27.2500 & $\begin{array}{l}\text { Glycosomal phosphoenolpyruvate } \\
\text { carboxykinase, putative }\end{array}$ & & N.D. \\
\hline Lin101E5 & -2.71 & $-1.4 \pm 0.5$ & 0.046 & 0 & 0 & $b$ & LinJ.35.5330 & Protein kinase, putative & & N.D. \\
\hline \multirow[t]{2}{*}{ Lin107B10 } & -2.22 & $-1.1 \pm 0.3$ & 0.003 & 0 & 0 & $b$ & LinJ.06.1110 & Deoxyribose phosphate aldolase, putative & + & $-7.3 \pm 0.6$ \\
\hline & & & & & & & LinJ.06.1120 & Hypothetical protein, conserved & & N.D. \\
\hline Lin115H5 & -2.37 & $-1.2 \pm 0.4$ & 0.034 & 0 & $3 e-136$ & $b$ & LinJ.03.0790 & $\begin{array}{l}\text { 6-phosphofructo-2-kinase/fructose-2,6- } \\
\text { diphosphatase, putative }\end{array}$ & & N.D. \\
\hline Lin123E6 & -2.11 & $-1.1 \pm 0.3$ & 0.019 & 0 & 0 & $b$ & LinJ.23.0980 & Actin-interacting protein & & N.D. \\
\hline Lin188B12 & -2.46 & $-1.3 \pm 0.1$ & 0.001 & 0 & 0 & $b$ & LinJ.31.3400 & Sodium stibogluconate-resistance protein & & N.D. \\
\hline Lin286D1 & -2.41 & $-1.3 \pm 0.4$ & 0.035 & 0 & 0 & $b$ & LinJ.08.1320 & Amastin-like protein & & N.D. \\
\hline Lin274G6 & -2.25 & $-1.2 \pm 0.4$ & 0.037 & 0 & 0 & $b$ & LinJ.08.0680/90 & Amastin-like protein & & N.D. \\
\hline Lin283F3 & -2.12 & $-1.1 \pm 0.3$ & 0.023 & 0 & 0 & $b$ & LinJ.15.0130 & ATP-dependent RNA helicase, putative & & N.D. \\
\hline Lin283H1 & -2.60 & $-1.4 \pm 0.2$ & 0.010 & 0 & 0 & $b$ & LinJ.21.1670 & $\begin{array}{l}\text { 2-oxoisovalerate dehydrogenase, } \\
\text { subunit a, putative }\end{array}$ & & N.D. \\
\hline Lin308D6 & -2.11 & $-1.1 \pm 0.1$ & 0.002 & 0 & 0 & $b$ & LinJ.11.0060 & Protein kinase, putative & & N.D. \\
\hline
\end{tabular}

Fold changes (up-regulation if $F>2$, over the dividing line, and down-regulation if $F<-2$, below the dividing line), base-two logarithmic scale $F$ and their SD, $\mathrm{p}$, e-values, clone definitions according to mapping outcomes $\mathrm{a}, \mathrm{b}$ and $\mathrm{c}$, Ids. and annotated functions in the $L$. infantum genome sequence and the qRT-PCR outcomes are provided. When a given clone overlaps with more than one annotation, only the clones checked for differential regulation using qRT-PCR and non-resolved clones overlapping with resolved clones containing a common gene appear in this table.

the up-regulation of this gene in amastigotes takes place independently of the source of promastigotes (culture or foregut of the sand fly). BLPL is not only essential for the branched-chain oxoacid dehydrogenase complex but also for the pyruvate dehydrogenase and the $\alpha$-ketoglutarate dehydrogenase complexes. As a difference with some genes involved in electron transport chain, none of the genes encoding proteins involved in pyruvate decarboxylation and the Krebs cycle are differentially regulated between Pro-Pper and amastigotes. The expression profile of BLPL may also be associated with fatty acid biosynthesis by the acetyl-CoA carboxylase. Up-regulation of the sphingolipid$\Delta^{4}$-desaturase cluster and the glycosylinositol phospholipid: galactofuranose (GIPL-galf) transferase gene in Pro-Pper (Table 2) suggests a possible increase of the demand of fatty acids in Pro-Pper. In fact, large amounts of unglycosylated inositolphosphoceramide molecules (IPC) [42] and GIPLs appear on the surface of the parasite and fatty acids are required for the biosynthesis of the corresponding lipid anchor. Palmitic acid is required for sphingosine biosynthesis, whereas the function of the GIPL-galf transferase is to add a galactofuranose residue to the exposed end of the molecule in GIPL-1 and close to the end in others, once the phospholipid anchor has been synthesized and fatty acids modified [43]. Sphingosine, ceramide and their phosphorylated derivatives are also signaling molecules as well as phospholipids, such as phosphatidic acid, lyso-phospholipids and phosphatidylinositol (PI). These molecules also participate in membrane trafficking and cytoskeleton remodeling. These facts also suggest an indirect role of BLPL and sphingolipid- $\Delta^{4}$-desaturase gene up-regulation in signaling in Pro-Pper, which is in agreement with the up-regulation of phosphatidylinositol 4-kinase (PI4K) (Table 2). However, these processes are activated by small $\mathrm{G}$ proteins at least in other eukaryotes [42] and the expression levels of the only annotated gene that encodes for this type of proteins in the L. infantum genome is over-expressed in amastigotes. As signaling pathways have not been yet elucidated in Leishmania spp. it is important to note that a correspondence in these processes between other eukaryotes, such as yeasts and mammals and the parasite may not be certain. Regarding vacuoles, genes encoding a vacuolar-associated membrane protein and a vacuolar proton translocating pyrophosphatase are up-regulated in Pro-Pper (Table 2), which may be related to an indirect membrane trafficking triggered by the up-regulation of sphingolipid- $\Delta 4$-desaturase and PI4K.

The up-regulation of the cysteine synthase (CS) and the cystathionine $\gamma$-lyase (CGL) genes in Pro-Pper (Table 2) suggests an increase of L-cysteine and, most likely, L-methionine biosynthesis. In addition, glutathione is synthesized from L-cysteine. This may be related to the over-expression of tryparedoxin peroxidase (TPXPx) that has been detected in Pro-Pper (Figure 4, Table 2). The pteridine transporter LinJ.06.1320 (PT) is also up-regulated at this stage compared to amastigotes. This difference was also found between cultured promastigotes and amastigote-like forms obtained by increasing the temperature from 27 to $37^{\circ} \mathrm{C}$ with and without a simultaneous $\mathrm{pH}$ decrease to 4.5 . Thus, a temperature increase is responsible for the down-regulation of this gene [16]. The 


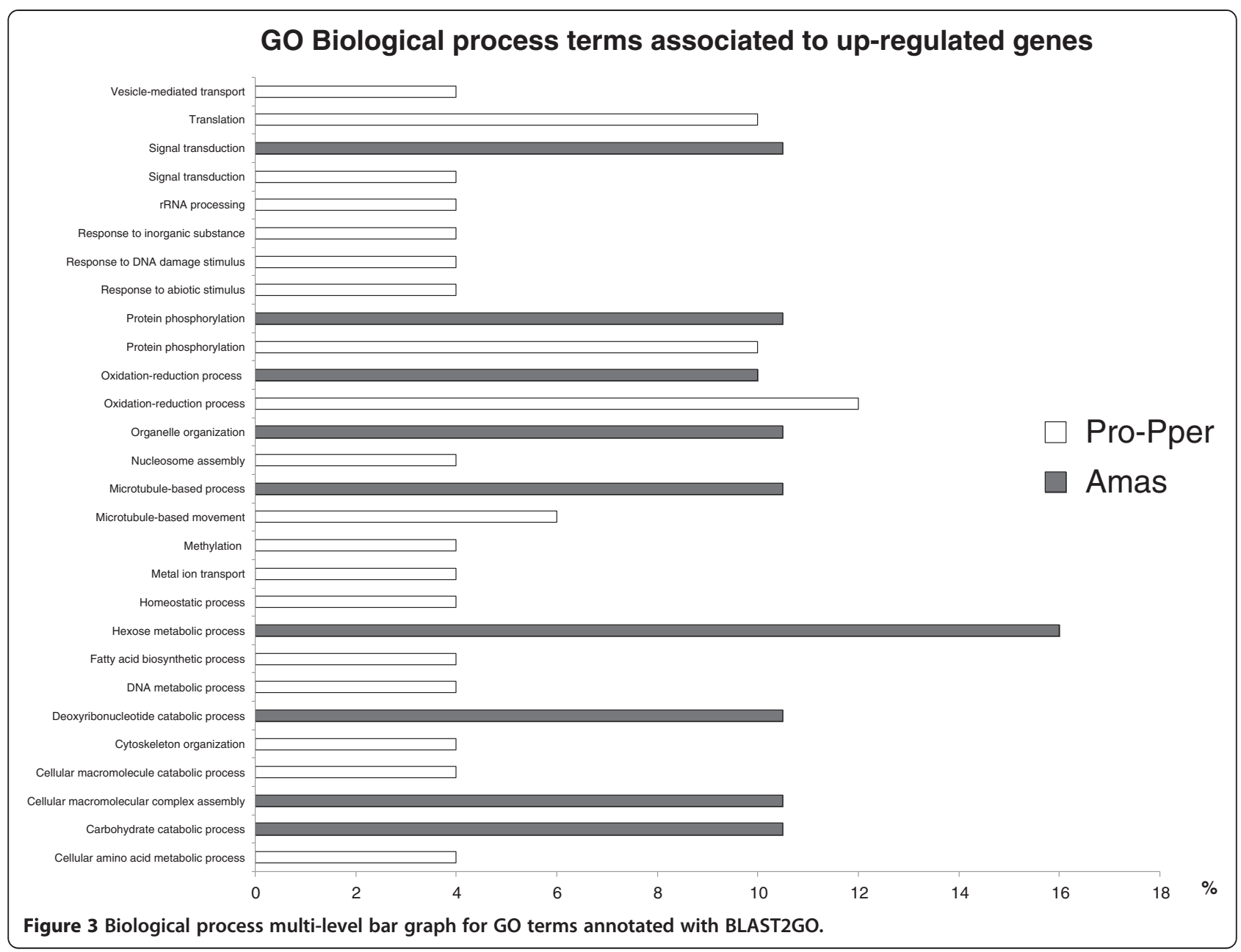

same expression profile of PT was found in L. mexicana [21], L. major [27] and L. infantum [16] amastigote-like forms and also L. infantum intracellular amastigotes [23]. Pterins are required for the biosynthesis of several amino acids such as methionine. This is most likely related to the up-regulation of CS and CGL, although an opposite expression pattern was found for the LinJ.10.0410 and LinJ.14.1440 genes, which encode PT isoforms in amastigote-like forms and amastigotes [16,23]. Nevertheless, these differences have not been found between Pro-Pper and amastigotes. Taken together, these data suggest that only PT LinJ.06.1320 is actually up-regulated in amastigotes in the natural life cycle of the parasite due to temperature increase and the other differences in transcript abundance may be related to the use of the culture model.

The gPEPCK expression profile may be also affected by serum in the culture medium. In fact, it is down-regulated under serum depletion (unpublished result), over-expressed in stationary compared to logarithmic phase promastigotes [23] and up-regulated in amastigotes with respect to Pro-Pper, but is not differentially expressed between amastigotes and cultured promastigotes. The inhibition of glycolysis in amastigotes may be carried out by fructose-2,6-diphosphate, as the 6-phosphofructo-2-kinase gene is up-regulated at this stage. Consequently, the role of gPEPCK up-regulation for monosaccharide supplies may be to accomplish the biosynthesis of glycoconjugates and/or sugar-derived metabolites. These findings are in agreement with the absence of monosaccharide sources in the environment of amastigotes, which has been previously reported [44]. In fact, it has been reported that promastigotes and amastigotes of Leishmania spp. can use amino acids as their major or only carbon source [45]. The upregulation of the deoxyribose phosphate aldolase EC 4.1.2.4 (DERA) gene, which is involved in deoxyribose phosphate catabolic processes (GO0046386), suggests that another possible source for amastigotes could be deoxynucleotide degradation, which may be taken from the environment. The products of the reaction catalyzed by DERA (acetaldehyde and glyceraldehydes-3-phosphate) are used as energy and carbon sources.

The glucose-6-phosphate $\mathrm{N}$-acetyltransferase gene (GNAT) is down-regulated in amastigotes not only with respect to cultured logarithmic and stationary phase 


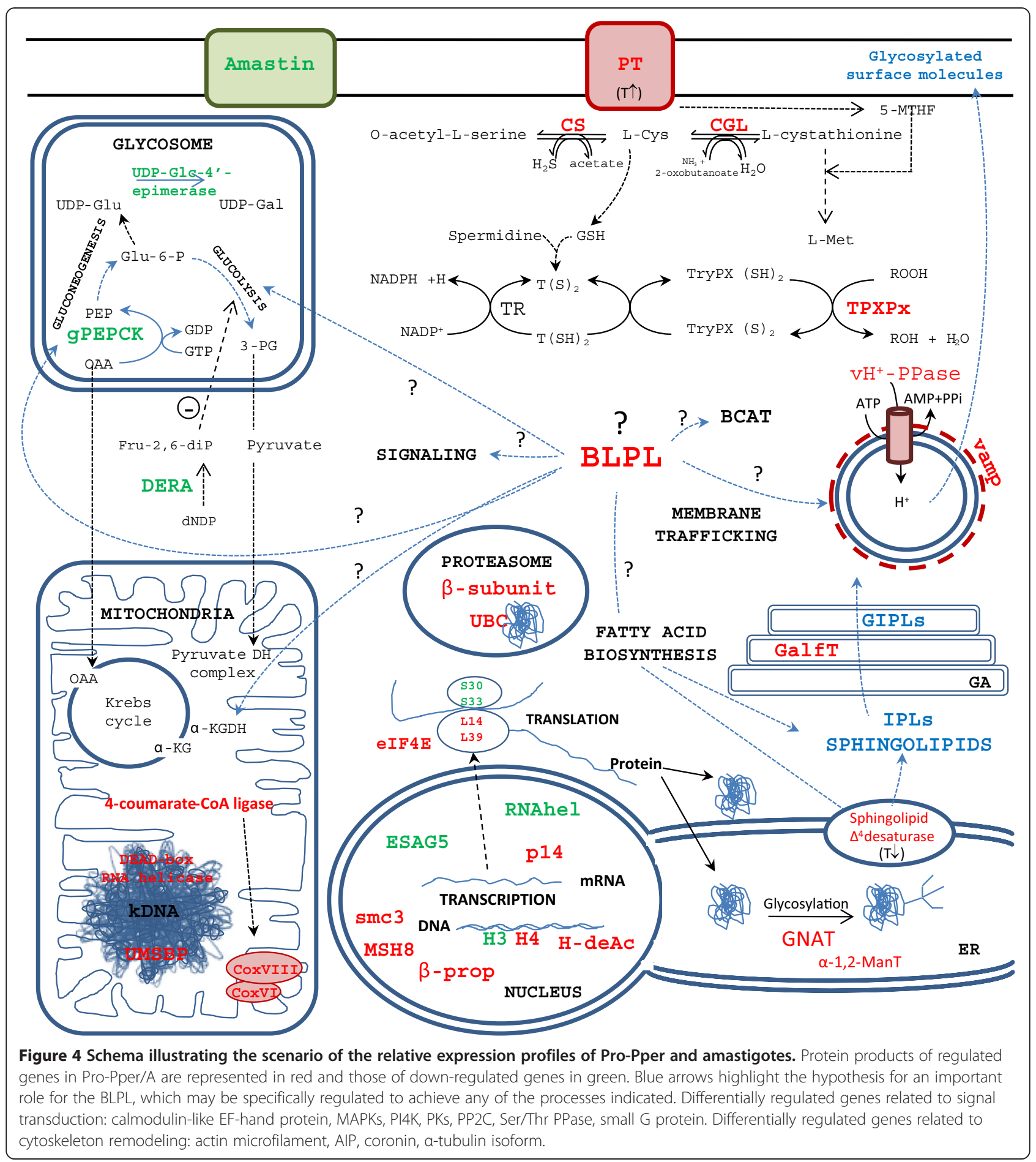

promastigotes [23] but also Pro-Pper. Although acidification alone leads to an increase of GNAT transcript abundance, the down-regulation of GNAT in amastigotes is due to the combined effect of temperature increase and $\mathrm{pH}$ decrease, as acidification does not lead to differentiation into amastigotes itself [16]. According to these data, GNAT transcript levels are less abundant in amastigotes than in promastigotes regardless of their origin (culture or foregut of the sand fly).

The following genes involved in gene expression regulation and intracellular signalling are up-regulated in Pro-Pper: eukaryotic initiation factor 4E (eIF4E), three ribosomal proteins (L39, S30 and S33), a small nuclear ribonucleoprotein, the pre-mRNA branch site 
p14 protein, a MAP kinase, a protein phosphatase $2 \mathrm{C}$ (PP2C), a Ser/Thr protein phosphatase, three protein kinases, a $\beta$-propeller protein, an EF hand-containing calmodulin-like protein, sphingolipid- $\Delta^{4}$-desaturase and the PI4K. Only the expression site-associated glycoprotein 5 (ESAG5) and an ATP-dependent DNA helicase genes are up-regulated in amastigotes. These findings suggest that a decrease in gene expression regulation and signaling activities take place in the differentiation process of promastigotes to amastigotes, which is in agreement with the lower up-regulation rate observed in amastigotes with respect to promastigotes independently of their origin (culture or foregut of the sand fly) (see [23] for cultured promastigotes and the next subsection for uncultured promastigotes). The pre-mRNA branch site $\mathrm{p} 14$ protein gene is also over-expressed in response to $\mathrm{pH}$ increase itself, providing additional evidence that this factor has limited influence on the differentiation process to amastigotes [16]. The amastin genes of cluster LinJ08.0680/ 0690/720/1320 are up-regulated in L. infantum amastigotes with respect to both cultured promastigotes $[15,16]$ and Pro-Pper (Table 2). Using the axenic culture model, it was found that the temperature increase, which is essential for differentiation of promastigotes to amastigotes, triggers the up-regulation of these genes [16]. These coincidences are not applicable for the surface sodium stibogluconate resistance protein (SbGRP) gene, as it shows the opposite expression profile between cultured promastigotes [23] and Pro-Pper (Table 2) with respect to amastigotes, a difference that may be due to the different environmental conditions inside the gut of the sand fly and axenic cultures. As for the microtubule cytoskeleton, this type of observations has also been made for the expression profile of the $\alpha$-tubulin LinJ.13.1450, which is up-regulated in amastigotes with respect to Pro-Pper but was previously found to be downregulated in amastigotes compared to $L$. donovani stationary phase promastigotes [29] and L. infantum stationary phase promastigotes [23]. Additionally, the OSM3 kinesin gene is up-regulated in Pro-Pper instead. The reason for the overexpression of the $\alpha$-tubulin gene in amastigotes is unknown as is a similar difference in the microfilament cytoskeleton, namely in an actin-interacting protein. Further investigations of these changes may reveal whether they are involved in the morphological changes these parasites undergo.

\section{More clues about promastigote pre-adaptation}

We described that the over-expression rate (number of up-regulated genes in a given stage or condition compared to the other one) is reduced in amastigotes with respect to cultured promastigotes of different Leishmania spp. [23], which supports the hypothesis of pre-adaptation of promastigotes, as stated by several authors [46,47]. In this case, the term pre-adaptation is understood to be the preparation in advance for intracellular survival once infection and differentiation to amastigotes occur. In fact, it has been reported that in some cases, amastigote-like forms are found within the population of metacyclic promastigotes located in the gut section anterior to the stomodeal valve of $P$. papatasi infected with $L$. major, which is most likely induced by respective slight temperature increase and a $\mathrm{pH}$ decrease after the female sand fly feeds [48]. This is in agreement with our previous findings about the effects of temperature and $\mathrm{pH}$ in the transcriptome during differentiation in L. infantum [16]. Tang et al. [49] measured the $\mathrm{pH}$ of the thoracic and abdominal mid gut of the sand fly Lutzomyia longipalpis concluding that before blood feeding, the $\mathrm{pH}$ is neutral in the thoracic mid gut and is alkaline in the abdominal mid gut and thereafter it diminishes to 6.8 or less. The $\mathrm{pH}$ in the parasitophorous vacuole of phagocytes of the mammalian host is between 4.5 and 5.5 and the temperature is about $37^{\circ} \mathrm{C}$ in the case of species responsible for visceral leishmaniasis. These findings support the hypothesis of pre-adaptation of promastigotes towards differentiation to amastigotes that has been previously proposed [46,47].

A binomial test has been performed for the set of differentially regulated genes between amastigotes and Pro-Pper (absolute frequencies in Table 1), and the outcome confirms a decrease of up-regulated genes in amastigotes with respect to Pro-Pper $(\mathrm{p}<0.0001)$, as it was reported using the culture model [23]. Overall, gene expression, signaling and response to stimulus, movement and response to stress are processes associated with up-regulated genes in Pro-Pper (Figure 3), suggesting a more active general metabolic status of promastigotes than amastigotes, which is consistent with the lower up-regulation rate in amastigotes and constitutes an additional evidence of the preadaptation hypothesis. Cellular component GO terms are in agreement, as ribosomes, the nucleolus, the nucleosome, the cytoskeleton and the proteasome are locations associated to some of the over-expressed genes. The genes involved in regulation of gene expression and intracellular signaling may be of special relevance. Thus, the expression profile of the biosynthetic gene sphingolipid- $\Delta^{4}$-desaturase gene may suggest an important role for IPC molecules in the differentiation of promastigotes to amastigotes, as they are involved in some of these important processes.

The GIPL-galf transferase is another gene up-regulated in Pro-Pper that may have an important role in pre-adaptation. In fact, McConville et al. [50] proposed that the GIPL molecules are present in all stages but are more abundant in amastigotes due to the relative decrease of glycoproteins, lipophosphoglycan (LPG) and proteophosphoglycan (PPG) on the amastigote surface and that the GIPLs protect other proteins of the plasma membrane against the lytic enzymes of the parasitophorous vacuole. Moreover, GIPL-1 plays a 
role in the interaction of promastigotes and amastigotes with macrophages [51]. The terminal galactofuranose residue it contains may be involved in macrophage recognition through a putative receptor that has been previously reported [51]. Therapeutic targets of the galactofuranose biosynthetic pathway have also been recently described in kinetoplastids causing leishmaniasis and Chagas disease [52]. As mentioned before, the up-regulation of the biotin/ lipoate ligase gene may be indirectly linked to the increase of GIPL biosynthesis in Pro-Pper.

The expression pattern of some of the genes of the amastin superfamily also provides a clue. Cultured stationary phase promastigotes show over-expression of amastin genes when compared to logarithmic phase amastigotes [23] but the highest levels are reached in amastigotes and cultured amastigote-like forms compared to cultured promastigotes $[15,16]$ or Pro-Pper. Although their role is unknown, these glycoproteins of the surface of amastigotes seem to be important for pathogenesis. In fact, Bolhassani et al. [53] reported partial protection in mice conferred by the amastin sequence fused to the VSP22 protein of herpes simplex virus 1 administered as a DNA vaccine.

\section{The influence of the promastigote culture model in} stage-specific gene regulation

The differential gene expression profiles between $L$. infantum promastigotes and amastigotes have been studied using cultured promastigotes either in logarithmic or stationary phase [23] and promastigotes isolated from the anterior gut of the sand fly $P$. perniciosus (this work).
The comparison between these analyses using promastigotes from culture and from the sand fly has been performed by Venn diagram and iterative hierarchical clustering (Figure 5, Additional file 5) and suggests that the culture conditions affect certain aspects of differentiation of promastigotes to amastigotes related to differential transcript abundance. In fact, only two genes (vesicleassociated membrane protein and sphingolipid- $\Delta 4$ desaturase, both down-regulated in amastigotes) show the same expression pattern between Pro-Pper and cultured logarithmic and stationary phase promastigotes. The number of similarities in the stage-specific expression profile during differentiation to amastigote between logarithmic and Pro-Pper is 11 genes, as well as for stationary phase vs. Pro-Pper. Some of these genes have known function: the histone H4 LinJ.36.0020 gene, the $\beta$-propeller protein LinJ.23.0040 and the histone deacetylase LinJ.08.1000, which differed between logarithmic phase promastigotes and Pro-Pper (all up-regulated with regard to amastigotes) and coxVI, GNAT, amastins of the LinJ.08.0680 cluster and the vacuolar proton-translocating pyrophosphatase, which differed between stationary phase promastigotes and Pro-Pper (all up-regulated with respect to amastigotes except the amastins). In addition, the profile of SbGRP and the $\alpha$-tubulin LinJ13.1450 is opposite between promastigotes in culture and in the anterior gut of the sand fly with respect to amastigotes. There are several coincidences with the outcome of the high-throughput iTRAQ-based proteome analysis described by Rosenzweig et al. [29], as 2-oxoisovalerate dehydrogenase, DERA and gPEPCK are up-regulated in $L$. donovani amastigotes vs.
A

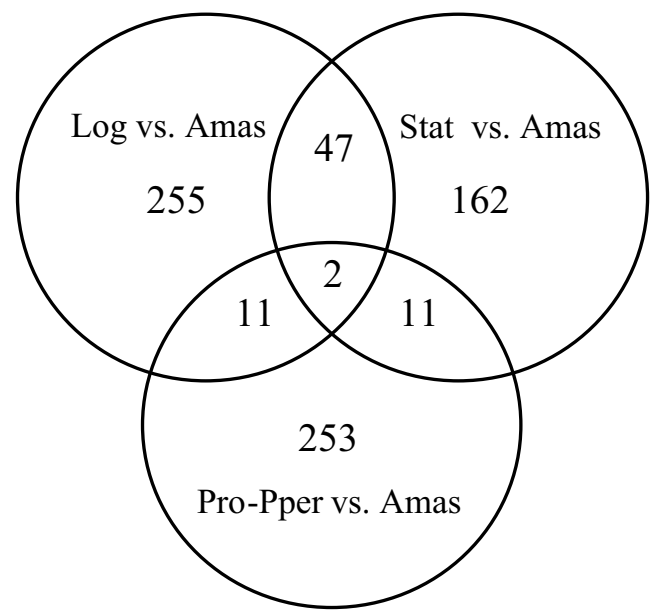

B

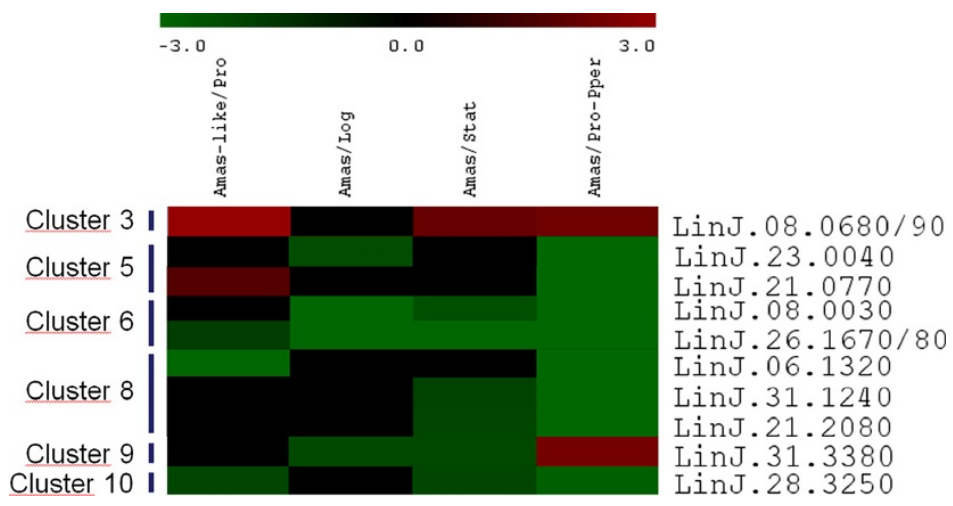

Figure 5 Comparison of the whole genome gene expression profile of amastigotes using Pro-Pper or cultured promastigotes as reference. The profiles of cultured logarithmic and stationary phase promastigotes compared with amastigotes have been published [23]. (A) Venn diagrams contrasting differential gene expression in L. infantum amastigotes depending on the origin of promastigotes. (B) Relative expression and classification (MEV analysis) in clusters of genes showing similar patterns or opposite expression profiles in amastigotes depending on the source of promastigotes (see a complete overview of the clustering analysis in Additional file 5). 
axenically cultured promastigotes, as well as in L. infantum vs. Pro-Pper, whereas the ATP-dependent RNA helicase LinJ.15.0130 shows an opposite pattern.

An illustrative example in the transcriptome profiles of sand fly-derived and cultured promastigotes during differentiation towards the amastigote stage is the up-regulation of the pre-mRNA branch site protein p14 gene in Pro-Pper with respect to amastigotes considered together with down-regulation after the treatment of promastigotes with $\mathrm{pH} 4.5$ [16]. $\mathrm{pH}$ is more acidic in a promastigote stationary culture (5.5-6.0) than in the thoracic mid gut and likely in the stomodeal valve (6.8 or lower according to Tang et al. [49]) and amastigotes are capable of withstanding $\mathrm{pH}$ values between 4.5 and 5.5. Thus, acidification turns the over-expression of the p14 gene in the slightly acidic environment of the $P$. perniciosus anterior gut into constant expression in more acidified stationary phase cultures and a further decrease of $\mathrm{pH}$ of the culture medium leads to under expression of this gene in forms of the parasite with differentially expressed transcriptome quite distinct from the natural promastigote and amastigote stages [16].

\section{Conclusions}

The differential expression profile of promastigotes to amastigotes, considering the initial and final time points (metacyclic promastigotes and amastigotes), is notably different when the source of metacyclic promastigotes is the foregut of the sand fly instead of axenic cultures. This finding suggests that using promastigote cultures may affect certain aspects of studying the parasite.

\section{Availability of the supporting data}

All data concerning the shotgun genomic DNA microarrays used and the hybridization procedure have been deposited in the GEO repository complying MIAME (http://www.ncbi. nlm.nih.gov/geo/query/acc.cgi?acc=GSE11269). Particular information about the sequences of primers and TaqMan probes used, hybridization controls in the microarray experiment, hypothetical proteins and analysis by gene clustering is available in the additional files that have been submitted along with this manuscript.

\section{Additional files}

Additional file 1: Primers and TaqMan-MGB probes used for qRT-PCR validation and the determination of differential expression in unresolved clones. Table S1. Sequences of GRT-PCR primers and probes.

Additional file 2: Clones that map with UTRs or less than $5 \%$ of length of an ORF. Table S2. Clones that do not fulfill the criteria specified in section the Methods section, Microarray hybridization and analysis of data subsection.
Additional file 3: Microarray controls. Table S3. The results of the Pro-Per/A cDNA-microarray hybridization analysis for positive and negative control spots.

Additional file 4: Hypothetical proteins. Table S4. Hypothetical proteins up-regulated in Pro-Pper/A. Table S5. Hypothetical proteins down-regulated in Pro-Pper/A.

Additional file 5: Overview of the MEV clustering analysis. Figure S1. Profile of clusters of genes differentially regulated in amastigotes.

\section{Abbreviations}

ABCE: ATP-binding cassette subfamily E; BLPL: Biotin/lipoate protein ligase; CGL: Cystathionine $\gamma$-lyase; cox: Cytochrome c oxidase; 4CL: 4-coumarateCoA ligase; CS: Cysteine synthase; DERA: Deoxyribose phosphate aldolase; elF4E: Eukaryotic initiation factor 4E; ESAG5: Expressed-site associated glycoprotein 5; GIPL: Glycosylinositolphospholipid;

GIPL-galfT: glycosylinositolphospholipid:galactofuranose transferase; GNAT: Glucose-6-phosphate N-acetyltransferase; gPEPCK: Glycosomal phosphoenolpyruvate carboxykinase; H-deAc: Histone deacetylase; HIFBS: Heat inactivated foetal bovine serum; IPL: Inositolphospholipid; IPC: Inositolphosphoceramide; a-KGDH: a-ketoglutarate dehydrogenase; KIVDH: a-ketoisovalerate dehydrogenase; LPG: Lipophosphoglycan; a-1,2-ManT: a-1,2-mannosyltransferase; MSH8: Mismatch repair protein 8; 5-MTHF: 5-methyltetrahydrofolate; OAA: Oxalacetate; NFQ: Non-fluorescent quencher; $\beta$-prop: $\beta$-propeller protein; Pl: Phosphatidylinositol;

PI4K: Phosphatidylinositol 4-kinase; PK: Protein kinase; PP2C: Protein phosphatase 2C; PPG: Proteophosphoglycan; PT: Pteridine transporter; qRT-PCR: Real time quantitative RT-PCR; sn-RNP: small nuclear ribonucleoprotein; SbGRP: Sodium stibogluconate resistance protein; TPXPX: Tryparedoxin peroxidase; TR: Trypanothione reductase; TryPX[SH]2: Reduced tryparedoxin; TryPX[S]2: Oxidized tryparedoxin; $\mathrm{T}[\mathrm{SH}]_{2}:$ Reduced trypanothione; $\mathrm{T}[\mathrm{S}]_{2}$ : Oxidized trypanothione; vamp: Vesicle-associated membrane protein; $\mathrm{VH}^{+}$-PPase: Vacuolar proton translocating pyrophosphatase; UBC: Ubiquitin-conjugating enzyme.

\section{Competing interests}

The authors declare that they have no competing interests.

\section{Authors' contributions}

All of the authors revised the manuscript thoroughly, made important contributions to the intellectual content of the manuscript and read and approved the final version of the manuscript. PA, AA and VL conceived and designed the experiment. MIJ and RM performed the sand fly infection and dissection procedures and prepared Pro-Pper. PA, AA, MIJ and RM prepared samples for microarray hybridization. PA and AA performed the microarray hybridizations and statistical analysis. MJG and MP sequenced the clone boundaries and performed the bioinformatic analysis. PA, AA and VL contributed to the thorough analysis and interpretation of the results and prepared the manuscript. PA and AA equally contributed to the coordination of the study.

\section{Acknowledgements}

We thank Alfredo Toraño, Mercedes Domínguez, Víctor Parro and Mercedes Moreno for their support. PA thanks CSIC for the I3P-BPD2003-1 grant and two contracts of employment for a position included in the A1 group (respectively developed from January 16th to July 23rd 2008 and from October 16th 2008 to April 15th 2009). AA thanks CSIC for the Ph.D. contract 5072160068 WOSC000077 within the A1 group. The cost of this work has been partially defrayed by the grants AGL2010-21806-C02-01 (Spanish Ministry of Science, MICINN), RICET (RETICS-FIS, FEDER) collaborative network grant and 050204100014 (Fundación Ramón Areces), OTT code 20100338.

\section{Author details}

'Laboratorio de Parasitología Molecular, Departamento de Microbiología Molecular y Biología de las Infecciones, Centro de Investigaciones Biologicas, Consejo Superior de Investigaciones Científicas, Calle Ramiro de Maeztu, 9. 28040 Madrid, Spain. ${ }^{2}$ Unidad de Secuenciación y Bioinformática, Centro de Astrobiología, Instituto Nacional de Técnica Aeroespacial "Esteban Terradas" and Consejo Superior de Investigaciones Científicas, Ctra. Ajalvir Km 4. 28850, Torrejón de Ardoz, Spain. ${ }^{3}$ Unidad de Entomología Médica, Servicio de Parasitología, Centro Nacional de Microbiología, Instituto de Salud Carlos III, 
Ctra. Majadahonda-Pozuelo s/n, 28220 Majadahonda, Spain. ${ }^{4}$ Current address: Centro Nacional de Investigaciones Cardiovasculares, Madrid, Spain.

Received: 9 January 2014 Accepted: 19 September 2014 Published: 3 October 2014

\section{References}

1. Desjeux P: Leishmaniasis. Public health aspects and control. Clin Dermatol 1996, 14(5):417-423.

2. WHO: Report of a Meeting of the WHO Expert Committee on the Control of Leishmaniases. Geneva: WHO Technical Report Serie; 2010

3. Pasquau F, Ena J, Sanchez R, Cuadrado JM, Amador C, Flores J, Benito C, Redondo C, Lacruz J, Abril V, Onofre J: Leishmaniasis as an opportunistic infection in HIV-infected patients: determinants of relapse and mortality in a collaborative study of 228 episodes in a Mediterreanean region. Eur J Clin Microbiol Infect Dis 2005, 24(6):411-418.

4. Cruz I, Nieto J, Moreno J, Canavate C, Desjeux P, Alvar J: Leishmania/HIV co-infections in the second decade. Indian J Med Res 2006, 123(3):357-388.

5. Arce A, Estirado A, Ordobas M, Sevilla S, Garcia N, Moratilla L, de la Fuente S, Martinez AM, Perez AM, Aranguez E, Iriso A, Sevillano O, Bernal J, Vilas F: Re-emergence of leishmaniasis in Spain: community outbreak in Madrid, Spain, 2009 to 2012. Euro Surveill 2013, 18(30):20546.

6. Molina R, Jimenez MI, Cruz I, Iriso A, Martin-Martin I, Sevillano O, Melero S, Bernal J: The hare (Lepus granatensis) as potential sylvatic reservoir of Leishmania infantum in Spain. Vet Parasitol 2012, 190(1-2):268-271.

7. Lucientes-Curdi J, Benito-de-Martin MI, Castillo-Hernandez JA, Orcajo-Teresa J: Seasonal dynamics of Larroussius species in Aragon (N.E. Spain). Parassitologia 1991, 33(Suppl):381-386.

8. Killick-Kendrick R: The biology and control of phlebotomine sand flies. Clin Dermatol 1999, 17(3):279-289.

9. Neal RA, Miles RA: Heated blood agar medium for the growth of Trypanosoma cruzi and some species of Leishmania. Nature 1963, 198:210-211.

10. Lemma A, Schiller EL: Extracellular cultivation of the leishmanial bodies of species belonging to the protozoan genus leishmania. Exp Parasitol 1964, 15:503-513.

11. Steiger RF, Steiger E: A defined medium for cultivating Leishmania donovani and L. braziliensis. J Parasitol 1976, 62(6):1010-1011.

12. Berens RL, Marr JJ: An easily prepared defined medium for cultivation of Leishmania donovani promastigotes. J Parasitol 1978, 64(1):160.

13. Zilberstein D: Physiological and Biochemical Aspects of Leishmania Development. In Leishmania After the Genome. Edited by Myler P, Fassel N. Norfolk: Caister Academic Press; 2008:107-122.

14. Zuckerman A, Lainson R: Leishmania. In Parasitic Protozoa. Edited by Kreier JP. New York: Academic Press; 1977:66-86

15. Rochette A, Raymond F, Corbeil J, Ouellette M, Papadopoulou B: Whole-genome comparative RNA expression profiling of axenic and intracellular amastigote forms of Leishmania infantum. Mol Biochem Parasitol 2009, 165(1):32-47.

16. Alcolea PJ, Alonso A, Gomez MJ, Sanchez-Gorostiaga A, Moreno-Paz M, Gonzalez-Pastor JE, Toraño A, Parro V, Larraga V: Temperature increase prevails over acidification in the gene expression modulation of amastigote differentiation in Leishmania infantum. BMC Genomics 2010, 11:31.

17. Akopyants NS, Matlib RS, Bukanova EN, Smeds MR, Brownstein BH, Stormo GD, Beverley SM: Expression profiling using random genomic DNA microarrays identifies differentially expressed genes associated with three major developmental stages of the protozoan parasite Leishmania major. Mol Biochem Parasitol 2004, 136(1):71-86.

18. Almeida R, Gilmartin BJ, McCann SH, Norrish A, Ivens AC, Lawson D, Levick MP, Smith DF, Dyall SD, Vetrie D, Freeman TC, Coulson RM, Sampaio I, Schneider H, Blackwell JM: Expression profiling of the Leishmania life cycle: CDNA arrays identify developmentally regulated genes present but not annotated in the genome. Mol Biochem Parasitol 2004, 136(1):87-100.

19. Saxena A, Lahav T, Holland N, Aggarwal G, Anupama A, Huang Y, Volpin $H$, Myler PJ, Zilberstein D: Analysis of the Leishmania donovani transcriptome reveals an ordered progression of transient and permanent changes in gene expression during differentiation. Mol Biochem Parasitol 2007, 152(1):53-65.

20. Saxena A, Worthey EA, Yan S, Leland A, Stuart KD, Myler PJ: Evaluation of differential gene expression in Leishmania major Friedlin procyclics and metacyclics using DNA microarray analysis. Mol Biochem Parasitol 2003, 129(1):103-114.

21. Holzer TR, McMaster WR, Forney JD: Expression profiling by whole-genome interspecies microarray hybridization reveals differential gene expression in procyclic promastigotes, lesion-derived amastigotes, and axenic amastigotes in Leishmania mexicana. Mol Biochem Parasitol 2006, 146(2):198-218.

22. Lahav $T$, Sivam D, Volpin H, Ronen M, Tsigankov P, Green A, Holland N Kuzyk M, Borchers C, Zilberstein D, Myler PJ: Multiple levels of gene regulation mediate differentiation of the intracellular pathogen Leishmania. FASEB J 2011, 25(2):515-525.

23. Alcolea PJ, Alonso A, Gomez MJ, Moreno I, Dominguez M, Parro V, Larraga V: Transcriptomics throughout the life cycle of Leishmania infantum: high down-regulation rate in the amastigote stage. Int J Parasito/ 2010, 40(13):1497-1516.

24. Alcolea PJ, Alonso A, Larraga V: Genome-wide gene expression profile induced by exposure to cadmium acetate in Leishmania infantum promastigotes. Int Microbiol 2011, 14(1):1-11.

25. Alcolea PJ, Alonso A, Larraga V: Proteome profiling of Leishmania infantum promastigotes. J Eukaryot Microbiol 2011, 58(4):352-358.

26. Alcolea PJ, Alonso A, Sanchez-Gorostiaga A, Moreno-Paz M, Gomez MJ Ramos I, Parro V, Larraga V: Genome-wide analysis reveals increased levels of transcripts related with infectivity in peanut lectin non-agglutinated promastigotes of Leishmania infantum. Genomics 2009, 93(6):551-564.

27. Leifso K, Cohen-Freue G, Dogra N, Murray A, McMaster WR: Genomic and proteomic expression analysis of Leishmania promastigote and amastigote life stages: the Leishmania genome is constitutively expressed. Mol Biochem Parasitol 2007, 152(1):35-46.

28. Rochette A, Raymond F, Ubeda JM, Smith M, Messier N, Boisvert S, Rigault P, Corbeil J, Ouellette M, Papadopoulou B: Genome-wide gene expression profiling analysis of Leishmania major and Leishmania infantum developmental stages reveals substantial differences between the two species. BMC Genomics 2008, 9:255.

29. Rosenzweig D, Smith D, Opperdoes F, Stern S, Olafson RW, Zilberstein D: Retooling Leishmania metabolism: from sand fly gut to human macrophage. Faseb J 2008, 22(2):590-602.

30. Sundstrom C, Nilsson K: Establishment and characterization of a human histiocytic lymphoma cell line (U-937). Int J Cancer 1976, 17(5):565-577.

31. Minta JO, Pambrun L: In vitro induction of cytologic and functional differentiation of the immature human monocytelike cell line U-937 with phorbol myristate acetate. Am J Pathol 1985, 119(1):111-126.

32. Hart DT, Vickerman $K$, Coombs GH: A quick, simple method for purifying Leishmania mexicana amastigotes in large numbers. Parasitology 1981, 82(Pt 3):345-355.

33. Molina R: Laboratory adaptation of an autochtonous colony of Phlebotomus perniciosus Newstead, 1911 (Diptera: Psychodidae). Res Rev Parasitol 1991, 51:87-89.

34. Jimenez M, Gonzalez E, Iriso A, Marco E, Alegret A, Fuster F, Molina R: Detection of Leishmania infantum and identification of blood meals in Phlebotomus perniciosus from a focus of human leishmaniasis in Madrid. Spain Parasitol Res 2013, 112(7):2453-2459.

35. Rastrojo A, Carrasco-Ramiro F, Martin D, Crespillo A, Reguera RM, Aguado B, Requena JM: The transcriptome of Leishmania major in the axenic promastigote stage: transcript annotation and relative expression levels by RNA-seq. BMC Genomics 2013, 14:223.

36. Conesa A, Gotz S, Garcia-Gomez JM, Terol J, Talon M, Robles M: Blast2GO: a universal tool for annotation, visualization and analysis in functional genomics research. Bioinformatics 2005, 21(18):3674-3676.

37. GeneDB. http://www.genedb.org/Homepage/Linfantum.

38. TriTrypDB. http://tritrypdb.org/tritrypdb/.

39. KEGG: Kyoto Encyclopedia of Genes and Genomes. http://www.genome. jp/kegg/.

40. Ivens AC, Peacock CS, Worthey EA, Murphy L, Aggarwal G, Berriman M, Sisk E, Rajandream MA, Adlem E, Aert R, Anupama A, Apostolou Z, Attipoe P, Bason N, Bauser C, Beck A, Beverley SM, Bianchettin G, Borzym K, Bothe G, Bruschi CV, Collins M, Cadag E, Ciarloni L, Clayton C, Coulson RM, Cronin A, Cruz AK, Davies RM, De Gaudenzi J, et al: The genome of the kinetoplastid parasite, Leishmania major. Science 2005, 309(5733):436-442.

41. Peacock CS, Seeger K, Harris D, Murphy L, Ruiz JC, Quail MA, Peters N, Adlem E, Tivey A, Aslett M, Kerhornou A, Ivens A, Fraser A, Rajandream MA Carver T, Norbertczak H, Chillingworth T, Hance Z, Jagels K, Moule S, 
Ormond D, Rutter S, Squares R, Whitehead S, Rabbinowitsch E, Arrowsmith C, White B, Thurston S, Bringaud F, Baldauf SL, et al: Comparative genomic analysis of three Leishmania species that cause diverse human disease. Nat Genet 2007, 39(7):839-847.

42. Zhang K, Barron T, Turco SJ, Beverley SM: The LPG1 gene family of Leishmania major. Mol Biochem Parasitol 2004, 136(1):11-23.

43. McConville MJ, Ferguson MAJ: The structure, biosynthesis and function of glycosylated phosphatidylinositols in the parasitic protozoa and higher eukaryotes. Biochem J 1993, 294:305-324.

44. Naderer T, Ellis MA, Sernee MF, De Souza DP, Curtis J, Handman E, McConville MJ: Virulence of Leishmania major in macrophages and mice requires the gluconeogenic enzyme fructose-1,6-bisphosphatase. Proc Natl Acad Sci U S A 2006, 103(14):5502-5507.

45. McConville MJ, De Souza DP, Saunders EC, Pyke J, Naderer T, Ellis MA, Sernee FM, Ralton JE, Likic VA: Analysis of the Leishmania Metabolome. In Leishmania After the Genome. Edited by Myler PJ, Fassel N. Norfolk: Caister Academic Press; 2008:75-106.

46. Sacks DL: Metacyclogenesis in Leishmania promastigotes. Exp Parasitol 1989, 69(1):100-103.

47. Depledge DP, Evans KJ, Ivens AC, Aziz N, Maroof A, Kaye PM, Smith DF: Comparative expression profiling of leishmania: modulation in gene expression between species and in different host genetic backgrounds. PLoS Negl Trop Dis 2009, 3(7):e476.

48. Anez N, Tang Y, Rojas A, Crisante G, Killick-Kendrick M, Killick-Kendrick R: Detection of amastigote-like forms in the valve of Phlebotomus papatasi infected with Leishmania major. Mem Inst Oswaldo Cruz 2003, 98(4):495-498.

49. Tang Y, Ward RD: Sugar feeding and fluid destination control in the phlebotomine sandfly Lutzomyia longipalpis (Diptera: Psychodidae). Med Vet Entomol 1998, 12(1):13-19.

50. McConville MJ, Mullin KA, llgoutz SC, Teasdale RD: Secretory pathway of trypanosomatid parasites. Microbiol Mol Biol Rev 2002, 66:122-154.

51. Suzuki E, Tanaka AK, Toledo MS, Takahashi HK, Straus AH: Role of beta-D-galactofuranose in Leishmania major macrophage invasion. Infect Immun 2002, 70(12):6592-6596.

52. Oppenheimer M, Valenciano AL, Sobrado P: Biosynthesis of galactofuranose in kinetoplastids: novel therapeutic targets for treating leishmaniasis and chagas' disease. Enzyme Res 2011, 2011:415976.

53. Bolhassani A, Gholami E, Zahedifard F, Moradin N, Parsi P, Doustdari F, Seyed N, Papadopoulou B, Rafati S: Leishmania major: protective capacity of DNA vaccine using amastin fused to HSV-1 VP22 and EGFP in BALB/C mice model. Exp Parasitol 2011, 128(1):9-17.

doi:10.1186/1471-2164-15-849

Cite this article as: Alcolea et al:: Stage-specific differential gene expression in Leishmania infantum: from the foregut of Phlebotomus perniciosus to the human phagocyte. BMC Genomics 2014 15:849.

\section{Submit your next manuscript to BioMed Central and take full advantage of:}

- Convenient online submission

- Thorough peer review

- No space constraints or color figure charges

- Immediate publication on acceptance

- Inclusion in PubMed, CAS, Scopus and Google Scholar

- Research which is freely available for redistribution 